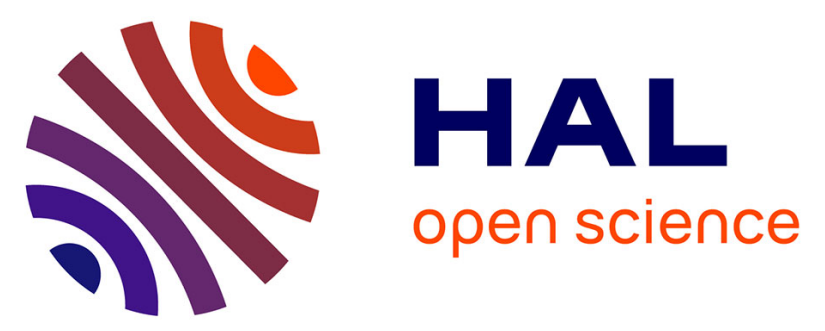

\title{
Compromise Based Evolutionary Multiobjective Optimization Algorithm for Multidisciplinary Optimization
}

\author{
Benoît Guédas, Xavier Gandibleux, Philippe Dépincé
}

\section{- To cite this version:}

Benoît Guédas, Xavier Gandibleux, Philippe Dépincé. Compromise Based Evolutionary Multiobjective Optimization Algorithm for Multidisciplinary Optimization. Yong Shi, Shouyang Wang, Gang Kou, Jyrki Wallenius. New State of MCDM in the 21st Century Selected Papers of the 20th International Conference on Multiple Criteria Decision Making 2009, Springer Berlin Heidelberg, pp.69-78, 2011, Lecture Notes in Economics and Mathematical Systems, 10.1007/978-3-642-19695-9_6 . hal00663702

\section{HAL Id: hal-00663702 \\ https://hal.science/hal-00663702}

Submitted on 27 Jan 2012

HAL is a multi-disciplinary open access archive for the deposit and dissemination of scientific research documents, whether they are published or not. The documents may come from teaching and research institutions in France or abroad, or from public or private research centers.
L'archive ouverte pluridisciplinaire HAL, est destinée au dépôt et à la diffusion de documents scientifiques de niveau recherche, publiés ou non, émanant des établissements d'enseignement et de recherche français ou étrangers, des laboratoires publics ou privés. 


\title{
Compromise based evolutionary multiobjective optimization algorithm for multidisciplinary optimization
}

\author{
Benoît Guédas Xavier Gandibleux Philippe Dépincé
}

\begin{abstract}
Multidisciplinary Design Optimization deals with engineering problems composed of several sub-problems -called disciplines- that can have antagonist goals and thus require to find compromise solutions. Moreover, the sub-problems are often multiobjective optimization problems. In this case, the compromise solutions between the disciplines are often considered as compromises between all objectives of the problem, which may be not relevant in this context. We propose two alternative definitions of the compromise between disciplines. Their implementations within the well-known NSGA-II algorithm are studied and results are discussed.
\end{abstract}

\section{Introduction}

The design and optimization of complex engineering systems, such as aircrafts, cars or boats, require the collaboration of many engineering teams from different disciplines. These problems are referred to as Multidisciplinary Design Optimization (MDO). This work deals with a class of MDO problems, where each discipline is a Multiobjective Optimization Problem (MOP - see [3] for notations and definitions). For instance, the design of a wing of an airplane involves two strongly coupled disciplines: aerodynamics and structure. Both may have several objectives to achieve. For example, the minimization of the drag and the maximization of the lift for aerodynamics and the minimization of the weight and of the deflection for the structure. The goal of such a MDO problem is to propose compromise solutions to the decision maker between the disciplines. In other contexts, compromises solutions have to be found between several possible scenarios (see for example [1] for an application to risk management). A compromise solution can be understood as a preferred solution for the decision problem.

\section{Context and goal}

Classical MDO methods [9] do not consider problems where the disciplines have multiple objectives each. Recently, Evolutionary Multiple Objective (EMO) optimization methods such as EM-MOGA [10], COSMOS [11] and others (see [7] for a description of these methods), were designed to solve optimization problems where each discipline has multiple objectives. But these approaches 
consider the problem as a unique MOP composed with all the objectives of the disciplines optimized simultaneously. Hence, the solutions found by those methods are the efficient solutions of the following problem:

$$
\min _{x \in X}\left(f_{1,1}(x), \ldots, f_{1, p_{1}}(x), \ldots, f_{q, 1}(x), \ldots, f_{q, p_{q}}(x)\right)
$$

where $q$ is the number of disciplines and $p_{i}$ the number of objectives of the $i^{\text {th }}$ discipline. The function $f_{i, j}$ represents the $j^{\text {th }}$ objective of the $i^{\text {th }}$ discipline. We will assume that all the disciplines are defined on the same decision space.

We will note $C_{0}$ the first compromise which consists in solving the MOP described eq. 1. Unfortunately, $C_{0}$ does not give relevant solutions in MDO problems, as illustrated by the follwing didactic example composed by two disciplines. Each discipline has two objectives to minimize: $f_{1,1}$ and $f_{1,2}$ for the first discipline and $f_{2,1}$ and $f_{2,2}$ for the second one. Let $f_{1}(x)=\left(f_{1,1}(x), f_{1,2}(x)\right)$ and $f_{2}(x)=\left(f_{2,1}(x), f_{2,2}(x)\right)$ be respectively the objective functions of the first and the second discipline, and $X=\left\{x_{a}, x_{b}, x_{c}\right\}$ the feasible set of solutions in the decision space. The performances of these solutions are reported in Table 1.

Table 1: The performances of the feasible solutions by $f_{1}\left(\right.$ resp. $\left.f_{2}\right)$ defined for Discipline 1 (resp. Discipline 2).

\begin{tabular}{|c||c|c|c|c|}
\hline \multicolumn{1}{|c||}{} & \multicolumn{2}{c|}{ Discipline 1 } & \multicolumn{2}{c|}{ Discipline 2 } \\
\hline$x$ & $f_{1,1}(x)$ & $f_{1,2}(x)$ & $f_{2,1}(x)$ & $f_{2,2}(x)$ \\
\hline \hline$x_{a}$ & 1 & 3 & 2 & 1 \\
\hline$x_{b}$ & 3 & 2 & 1 & 3 \\
\hline$x_{c}$ & 2 & 1 & 3 & 2 \\
\hline
\end{tabular}
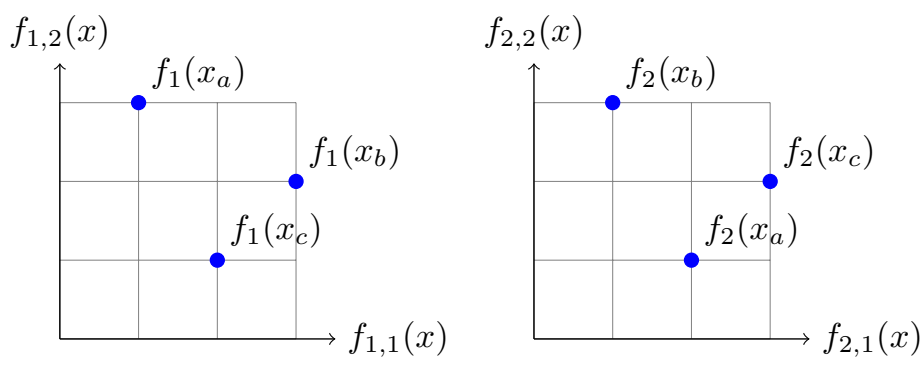

Figure 1: The objective spaces of the two disciplines of the didactic minimization problem: discipline 1 is on the left and discipline 2 on the right.

Considering independently the disciplines, we have two efficient solutions by discipline (see Fig. 1): $x_{a}$ and $x_{c}$ in the first discipline, and $x_{a}$ and $x_{b}$ in the second one. As $x_{a}$ is an efficient solution in both disciplines, $x_{a}$ is expected to be the only efficient compromise solution of this problem and reported as the unique output of the algorithm. But if we consider the four objectives problem (eq. 1), all the feasible solutions are efficient because their 4-dimensions performance vectors are mutually non-dominated: the $C_{0}$ compromise solution set is thus $X$. Indeed, solving the MOP can be interpreted as finding solutions between 
Table 2: Ranks of the points in each discipline: $r_{1}(x)$ (resp. $r_{2}(x)$ ) is the rank of $x$ in the discipline 1 (resp. discipline 2).

\begin{tabular}{|c|c|c|}
\hline$x$ & $r_{1}(x)$ & $r_{2}(x)$ \\
\hline$x_{a}$ & 1 & 1 \\
\hline$x_{b}$ & 2 & 1 \\
\hline$x_{c}$ & 1 & 2 \\
\hline
\end{tabular}

objectives without taking into account the grouping of the objectives within the disciplines. For instance, $x_{a}$ would be preferred over $x_{b}$ in the first discipline and over $x_{c}$ in the second one but this information is missing in the $C_{0}$ compromise. We propose two compromises which are more relevant in this context: $C_{1}$ and $\mathrm{C}_{2}$.

\section{Computing a compromise}

\section{1 $C_{1}$ : Ordering the solutions in disciplines}

The first idea we propose - also presented in [8] - is to transform each disciplinary MOP into a single objective using a measure of solutions quality inside each discipline.

Ranking procedures are frequently used in multiobjective genetic algorithms for evaluating the fitness of the solutions. Goldberg [6] first introduced the rank to bias the selection operator based on the evaluations of the objective function. This idea has then been used by Srinivas and Deb [12] in the NSGA algorithm in order to sort the individuals for a multiobjective problem. Other ranking procedures have been proposed for multiobjective genetic algorithms, such as the one proposed by Fonseca and Fleming [5]. Definition 1 presents the rank as it is defined in ordered sets theory. This definition is the same as the one used by Srinivas and Deb.

Definition Let $O=(E, \leq)$ be an ordered set. For $e \in E$, the rank $r(e)$ of $e$ is defined as follows:

- $r(e)=1$, if $e$ is a minimum.

- $r(e)=n$, if the elements of rank $<n$ have been assigned and $e$ is a minimum in the ordered set $P \backslash\{x \in P: r(x)<n\}$.

The compromise $C_{1}$ consists in using the rank as the only objective of each discipline. The corresponding multidisciplinary optimization problem can be formulated as the following MOP:

$$
\min _{x \in X}\left(r_{1}(x), \ldots, r_{q}(x)\right)
$$

where $r_{i}(x)$ represents the rank of $x$ in the $i^{\text {th }}$ discipline. The efficient solutions of eq. 2 are the $C_{1}$-compromise solutions.

For instance, in the didactic example introduced in the previous section (Table 1, Fig. 1), each point is ranked in each discipline separately. The results 
are presented in Tab 2. Considering these ranks as performances of the solutions in each discipline, we can define a compromise solution as a non-dominated ranks vector. There is only one non-dominated rank vector $(1,1)$, so the only $C_{1}$-compromise solution to this problem is $x_{a}$.

Unfortunately, a compromise based on ranks has an important drawback if implemented within an EMO algorithm because of its stochastic behavior: the order relation between two performance vectors depends on the spread of the performance vectors of the other individuals in the population. Thus, as the population evolves, the performance vectors move. The Fig. 2 shows that on the first chart, $f_{i}\left(x_{a}\right)$ is ranked 1 and $f_{i}\left(x_{c}\right)$ is ranked 2, whereas $f_{i}\left(x_{a}\right)$ is ranked 3 and $f_{i}\left(x_{c}\right)$ is ranked 2 on the second chart. Between the first and the second chart, only two points, $f_{i}\left(x_{d}\right)$ and $f_{i}\left(x_{e}\right)$ have been added, but it results in a reverse of the order of $f_{i}\left(x_{a}\right)$ and $f_{i}\left(x_{c}\right): f_{i}\left(x_{c}\right)$ was greater than $f_{i}\left(x_{a}\right)$ on the first chart whereas it is less than $f_{i}\left(x_{a}\right)$ on the second one.
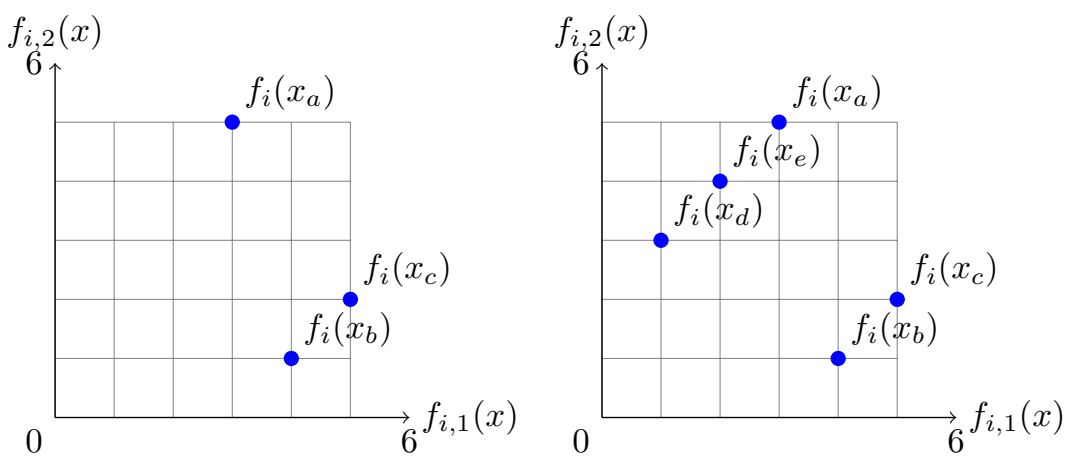

Figure 2: Using the rank to define an order relation between elements of a discipline $i$, the order relation depends on the other elements: the rank of $f_{i}\left(x_{a}\right)$ is less than the rank of $f_{i}\left(x_{c}\right)$ in the left chart whereas this relation is reversed in the right chart.

\section{$3.2 C_{2}$ : Taking into account incomparability in the disci- plines}

Another approach is to take into account the incomparabilities in the dominance relation: an element $f(x)$ dominates $f\left(x^{\prime}\right)$ if $f(x)$ dominates $f\left(x^{\prime}\right)$ in at least one discipline, and $f\left(x^{\prime}\right)$ never dominates $f(x)$ :

$$
f(x) \preceq f\left(x^{\prime}\right) \Longleftrightarrow \begin{cases}\exists i \in\{1, \ldots, n\} & f_{i}(x) \leq_{i} f_{i}\left(x^{\prime}\right) \\ \nexists j \in\{1, \ldots, n\} & f_{j}\left(x^{\prime}\right) \leq_{j} f_{j}(x)\end{cases}
$$

where $\leq_{i}$ is the componentwise order in the discipline $i$, and $\leq_{j}$ is the Paretodominance relation in the discipline $j$. Let call $C_{2}$ the compromise such that the compromise solutions are such that their performance vectors in each disciplines are non-dominated according to the transitive closure of the $\preceq$ relation.

In the didactic example presented Fig. 1, let $f(x)=\left(f_{1}(x), f_{2}(x)\right)$. The point $f\left(x_{a}\right)$ dominates $f\left(x_{c}\right)$ and $f\left(x_{c}\right)$ dominates $f\left(x_{b}\right)$ in one discipline each, 
and there are no other domination relations. So we have $f\left(x_{a}\right) \preceq f\left(x_{c}\right) \preceq f\left(x_{b}\right)$. Thus, $x_{a}$ is the only solution to this problem. On this example, the solutions of $C_{2}$ are equivalent to $C_{1}$. Moreover, this compromise is expected to be less sensitive when implemented within an EMO algorithm, because it does not introduce new order relations in the disciplines depending on the spread of the population.

\section{EMO-MDO algorithm}

The proposed algorithm, called EMO-MDO (Algorithm 1), is a generalization of the NSGA-II algorithm [2]. Its particularity lies in the ranking procedure which can be computed on any preordered set ${ }^{1}$, and not only the performance vectors of the individuals. It allows to modelize different kind of compromises such as $C_{0}, C_{1}$ and $C_{2}$. In particular, EMO-MDO used with $C_{0}$ is identical to NSGA-II. It also shares its genetic operators: real coded genes, SBX crossover (crossover routine), polynomial mutation (mutation routine), crowded binary constrained tournament selection (selection routine) and non-constrain-dominated sorting procedure with crowding (generation routine) [2].

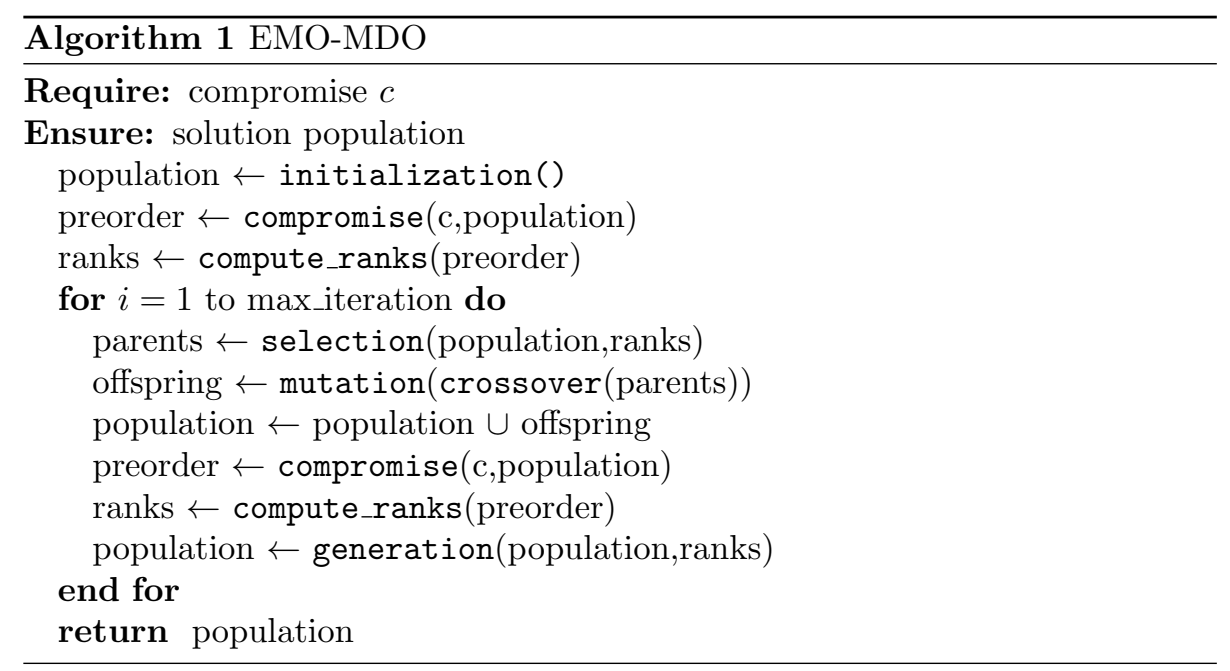

The initial population of size $N$ is created from solutions randomly picked from the feasible set. The preorder between the individuals is then computed by the compromise function according to the population pop and the compromise $c \in\left\{C_{0}, C_{1}, C_{2}\right\}$. This preorder is used to compute the ranks of the individuals in the population. A parents population is selected from pop according to their ranks. Crossovers and mutations are performed to create an offspring population. The offspring is then added to the current population pop to create a $2 \times N$ population on which the compromise relation is computed. The generation function selects the new individuals which will belong to the next generation.

\footnotetext{
${ }^{1}$ Ordered sets which are not necessarily antisymmetric.
} 


\section{$5 \quad$ Numerical experiments}

A series of experiments were performed on the EMO-MDO in order to study its behavior on $C_{0}, C_{1}$ and $C_{2}$ compromises.

\section{$5.1 \quad$ Test problem}

This test problem comes from Engau and Wiecek [4]. It is composed of two biobjective disciplines defined on the feasible set $X$. Here, all the feasible solutions are efficient for $C_{0}$ compromise.

$$
\begin{aligned}
& D_{1}\left\{\begin{array}{l}
\min _{\left(x_{1}, x_{2}\right) \in X} f_{1,1}\left(x_{1}, x_{2}\right)=\left(x_{1}-2\right)^{2}+\left(x_{2}-1\right)^{2} \\
\min _{\left(x_{1}, x_{2}\right) \in X} f_{1,2}\left(x_{1}, x_{2}\right)=x_{1}^{2}+\left(x_{2}-3\right)^{2}
\end{array}\right. \\
& D_{2}\left\{\begin{array}{l}
\min _{\left(x_{1}, x_{2}\right) \in X} f_{2,1}\left(x_{1}, x_{2}\right)=\left(x_{1}-1\right)^{2}+\left(x_{2}+1\right)^{2} \\
\min _{\left(x_{1}, x_{2}\right) \in X} f_{2,2}\left(x_{1}, x_{2}\right)=\left(x_{1}+1\right)^{2}+\left(x_{2}-1\right)^{2}
\end{array}\right.
\end{aligned}
$$

with $X=\left\{\left(x_{1}, x_{2}\right) \in \mathbb{R}^{2}: x_{1}^{2}-x_{2} \leq 0, x_{1}+x_{2}-2 \leq 0,-x_{1} \leq 0\right\}$

\subsection{Test protocol}

Two reference sets of solutions are computed by filtering the solutions satisfying the compromises from a sampling of the feasible set of decision variables. The first sampling corresponds to a uniform discretization of the feasible set, whereas the second sampling is a simple random sampling. For each test, 300 solutions are evaluated.

The three compromises have been compared with the filtering methods and the EMO-MDO algorithm. The latter has been performed in two conditions: with or without the phenotypic diversity procedure enabled (crowding) in the selection and the generation functions.

The EMO-MDO algorithm as been run with the following combinations of population sizes and iterations numbers: 10x50, 20x15, 20x20, 50x50 and $50 \times 500$. Three simulations of each combination have been done to verify the stability of the algorithm.

From these experiments, we want to compare the solutions found by the genetic algorithms to the reference solutions, and to compare $C_{1}$ and $C_{2}$ compromises to $C_{0}$. As far as we know, there is no quality measure of the solutions of such problems as they exist in the field of evolutionary multiobjective genetic algorithms.

\subsection{Results and discussion}

The crossover and mutation probabilities are respectively 0.8 and 0.2 . The $\eta$ and $\sigma$ parameters for the SBX crossover and mutation are respectively 2 and 4 . Other set of parameters have been tested and they produce comparable results 
Discipline 1
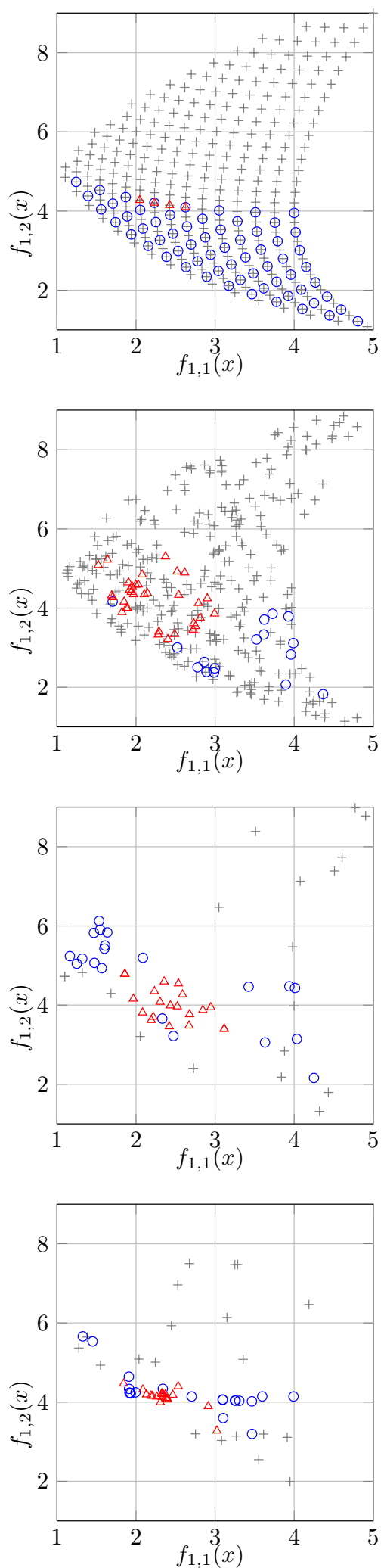

Discipline 2

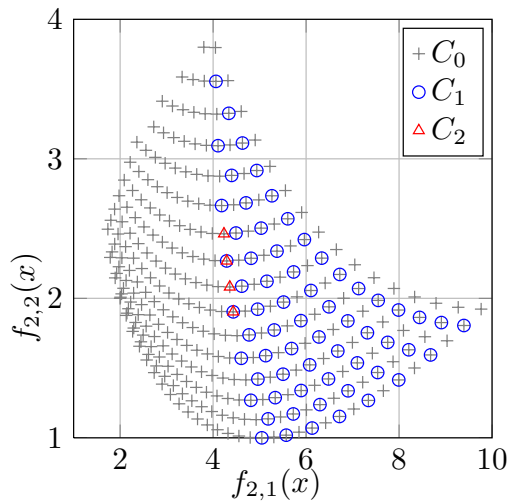

(a)

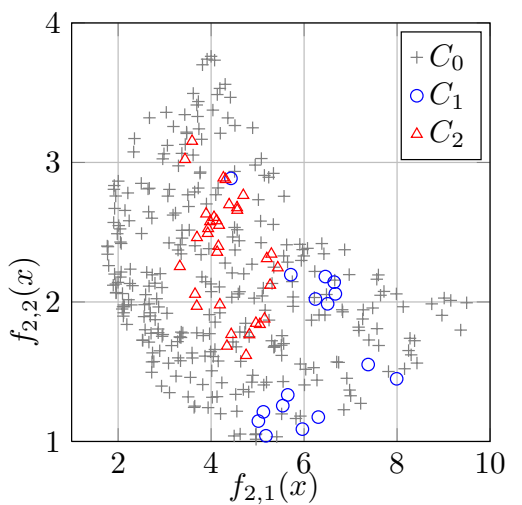

(b)

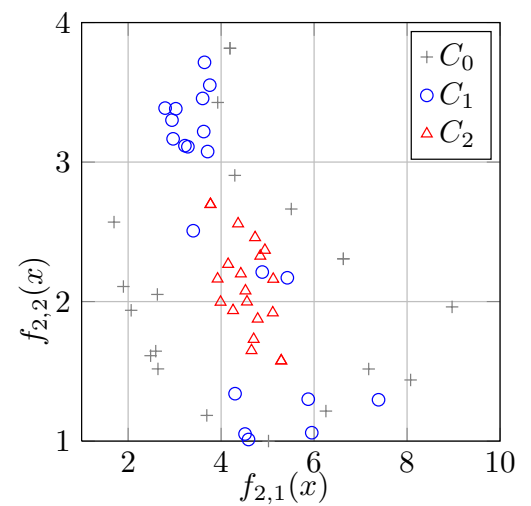

(c)

(d)

Figure 3: Results of the test problem using compromise $C_{0}, C_{1}$ and $C_{2}$ with filtering on uniform sampling (a), and simple random sampling (b) of 300 points. Results with EMO-MDO with phenotypic distance enabled (c), and disabled (d). Population size is 20, and 15 iterations were performed. 
- We observe that the compromise solutions found with $C_{1}$ with the uniform sampling (figure 3-a) are uniformly distributed in an area approximately equal to the half of the image of the feasible set.

- A random sampling of the feasible set (figure 3-b) does not give the same results: the compromise solutions still belong to the same area but are not spread uniformly. This shows that the solutions of the compromise $C_{1}$ are dependent to the spread of the population as stated in section 3 .

- The $C_{2}$ solutions are more largely spread with the simple random sampling than the uniform discretization (figure $3-\mathrm{a}, \mathrm{b}$ ). Nevertheless, the solutions are in the same area, and this spread decrease as the sampling size increase.

- The EMO-MDO algorithm did not converge to the expected area when used with $C_{1}$ (figure 3 -c,d). This is more salient when phenotypic diversity is disabled (figure $3-\mathrm{d}$ ).

- The EMO-MDO algorithm converged to the expected area when used with $C_{2}$ (figure 3 -c,d).

- The phenotypic diversity of NSGA-II is not adapted to this kind of problem: many solutions have the same performances within a discipline (figure 3-c). Nevertheless, phenotypic diversity still improves the solutions: they are less diversifyied when disabled (figure 3-d).

We also observed that the compromise $C_{2}$ is less sensitive to the algorithm parameters than the compromise $C_{1}$.

\section{Conclusions and on-going work}

MDO problems are often composed of disciplines which have several objectives each. The compromise solutions are then often defined as the efficient solutions of the MOP $\left(C_{0}\right)$. This can be interpreted as a compromise between the objectives instead of a compromise between the disciplines.

We propose a first compromise $\left(C_{1}\right)$ based on the preferences of the disciplines using the ranks of the solutions. Unfortunately, this does not suit to an EMO algorithm because ranks will not change uniformly as the population evolves. To overcome this problem, we propose another compromise $\left(C_{2}\right)$ allowing a non total order of the preferences of the disciplines. $C_{0} C_{1}$ and $C_{2}$ have been implemented into an EMO algorithm. $C_{2}$ gives satisfying results and is less sensitive to the algorithm parameters than $C_{1}$.

The proposed compromises have been defined on a simplified class of MDO problems without local variables and coupling functions between disciplines. Our future research will extend the compromises to more complex problems.

Acknowledgments The authors would like to thank the regional council of the Pays de la Loire (France), MILES project, for their support of this research. 


\section{References}

[1] Better M., Glover F., Kochenberger G., Wang H.: Simulation optimization: application in risk management. International Journal of Information Technology \& Decision Making 7(4):571-587 (2008).

[2] Deb K.: Multi-objective optimization using evolutionary algorithms. Wiley (2001). 518 p.

[3] Ehrgott M.: Multicriteria optimization. Springer, Berlin Heidelberg, 2nd edition (2005). $323 \mathrm{p}$.

[4] Engau A., Wiecek M.M.: 2D decision-making for multicriteria design optimization. Structural and Multidisciplinary Optimization 34(4):301-315 (2007).

[5] Fonseca C., Fleming P.: Genetic algorithms for multiobjective optimization: formulation discussion and generalization. In : Proceedings of the 5th International Conference on Genetic Algorithms, pp. 416-423, San Francisco, CA, USA (1993).

[6] Goldberg D.E.: Genetic algorithms in search, optimization and machine learning. Addison-Wesley, Reading, Massachusetts (1989). 432 p.

[7] Guédas B.: Proposition de compromis pour le calcul de solutions préférées à l'aide d'un algorithme évolutionnaire multiobjectif en optimisation multidisciplinaire. Ph.D. Thesis, École Centrale de Nantes, France (2010).

[8] Guédas B., Dépincé P., Gandibleux X.: Vers une notion de compromis en optimisation multidisciplinaire multiobjectif. In: Book of abstracts of the ROADEF 2009 Conf., Nancy, France (2009).

[9] Tosserams S., Etman L.F.P., Rooda J.E.: A classification of methods for distributed system optimization based on formulation structure. Structural and Multidisciplinary Optimization 39(5):503-517 (2009).

[10] Gunawan S., Farhang-Mehr A., Azarm S.: Multi-level multi-objective genetic algorithm using entropy to preserve diversity. In: Fonseca C.M., Fleming P., Zitzler E., Deb K., Thiele L. (eds) Evolutionary Multicriterion Optimization, LNCS 2632, pp. 148-161 (2003) . Springer, Berlin Heidelberg.

[11] Rabeau S., Dépincé P., and Bennis F.: Collaborative optimization of complex systems: a multidisciplinary approach. International Journal on Interactive Design and Manufacturing 1(4):209-218 (2007).

[12] Srinivas N., Deb K.: Multiobjective optimization using nondominated sorting in genetic algorithm. Evolutionary Computation 2(3):221-248 (1994). 